\title{
Prevalência de excesso de peso entre estudantes de ensino fundamental de escola pública e privada em Sorocaba, São Paulo, Brasil
}

\author{
Prevalence of overweight among students of public and private elementary and middle schools in \\ Sorocaba, São Paulo, Brazil
}

\section{Prevalencia de sobrepeso entre los estudiantes de primaria en escuelas públicas y privadas en Sorocaba, São Paulo, Brasil}

Inês Maria Pardo. Pontifícia Universidade Católica de São Paulo (PUC-SP). doctorpardo@hotmail.com (Autora correspondente) Mariana Pacifico Mercadante. Pontifícia Universidade Católica de São Paulo (PUC-SP). marianapmercadante@gmail.com Mayara Franco Zanatta. Pontifícia Universidade Católica de São Paulo (PUC-SP). my.lowin@hotmail.com Valéria Cristina Santucci Ramos. Pontifícia Universidade Católica de São Paulo (PUC-SP). vcsr@ig.com.br Sandra Dantas Nascimento. Pontifícia Universidade Católica de São Paulo (PUC-SP). sandradantas@uol.com.br José Eduardo Bueno Miranda. Pontifícia Universidade Católica de São Paulo (PUC-SP). jegbmiranda@uol.com.br

\section{Resumo}

Objetivo: Este estudo teve como objetivo avaliar a prevalência de sobrepeso e obesidade em um grupo de estudantes e investigar se há correlação entre IMC (índice de massa corporal) e renda familiar. Métodos: Estudo tipo transversal, realizado com 213 estudantes do ensino fundamental de escola pública (EPU) e privada (EPR), com idade entre 10 e 14 anos, aprovado pelo comitê de ética local e mediante assinatura do termo de consentimento livre e esclarecido pelos estudantes e os responsáveis por eles. Os estudantes foram pesados e medidos e o IMC foi calculado; os dados foram avaliados com base nos gráficos da OMS para IMC conforme 0 sexo. Os estudantes responderam ao questionário socioeconômico segundo 0 critério da ABEP. A análise dos dados foi realizada usando o programa SPSS. Foi adotado nível de significância de 5\%. Resultados: Prevalência global de excesso de peso (sobrepeso) na amostra estudada foi de 41,3\%. Na comparação dos resultados isolados, a frequência de excesso de peso foi maior em alunos da escola particular (47,3\%) do que nos da escola pública (32,1\%), com significância estatística ( $p=0,03)$. A frequência de excesso de peso nos meninos da EPR foi maior do que nos da EPU (23,3\% versus 14,3\%; $p=0,05)$. Nas meninas, verificou-se a mesma tendência, sem, todavia, significância estatística (24,0\% versus 17,9\%; $p=0,18)$. Houve correlação positiva entre IMC e renda familiar ( $r=0,14, p=0,04)$. Conclusão: Cerca de $40 \%$ dos estudantes entrevistados apresentaram excesso de peso, com prevalência maior entre os meninos da escola particular. É relevante o desenvolvimento de estratégias de prevenção da obesidade em todos os níveis da sociedade, pois há potenciais riscos à saúde na vida adulta.

\section{Abstract}

Objective: The objectives of this study were to evaluate the prevalence of overweight and obesity in a sample of students and investigate the correlation between BMI (body mass index) and family income. Methods: A cross-sectional study conducted with 213 primary school students of public (PUS) and private (PRS) schools, aged between 10 and 14 years. This study was approved by the local Ethics Committee, and students and parents who were willing to participate could only do it by signing a consent form. Students were weighed and measured, and the BMI was calculated, the resulting data were evaluated according to the WHO BMI charts related to sex. The students answered a questionnaire on socioeconomic status according to the 'ABEP' charts. Data analysis was performed using SPSS, with 5\% significance level. Results: The total prevalence of overweight in the sample studied was of $41.3 \%$. Comparing the individual results, the frequency of overweight was higher for private school (47.3\%) than for public school $(32.1 \%)$ children, with statistical significance $(p=0.03)$. The frequency of overweight among PRS boys was higher than among the PUS boys $(23.3 \%$ versus $14.3 \%, p=0.05)$. In girls, the same trend was observed, but with no statistical significance $(24.0 \%$ versus $17.9 \%, p=0.18)$. There was a positive correlation between BMl and family income $(r=0.14, p=0.04)$. Conclusion: About $40 \%$ of the students who participated in the study were overweight, with higher prevalence among private school boys. It is important to establish strategies to prevent obesity at all levels of society, considering the potential health risks for those overweight children when they reach adulthood.

\section{Palavras-chave: \\ IMC}

Índice de Massa Corporal

Adolescentes

Classe Social

\section{Keywords:}

BMI Body Mass Index Adolescents Social Class 


\section{Resumen}

Objetivo: Los objetivos de esto estudio fueron evaluar la prevalencia del sobrepeso y obesidad en una muestra de estudiantes e investigar si hay correlación entre el IMC (índice de masa corporal) y el ingreso familiar. Métodos: Estudio transversal realizado en 213 estudiantes del primaria de escuela pública (EPU) y privada (EPR), con edades comprendidas entre 10 y 14 años. Este estudio fue aprobado por el Comité de Ética local, mediante la firma de un consentimiento informado, libre y claro, por los estudiantes y sus padres. Los estudiantes fueron pesados y medidos y se calculó el IMC; Ios datos resultantes fueron evaluados de acuerdo con los gráficos de la OMS para IMC para hombres y mujeres. Los estudiantes respondieron a un cuestionario sobre la situación socioeconómica a discreción de ABEP. El análisis de los datos se realizó mediante el programa SPSS, con el nivel de significancia de 5\%. Resultados y discusión: La prevalencia global de sobrepeso en la muestra estudiada fue de 41,3\%. Comparando los resultados de forma aislada, la frecuencia de sobrepeso fue significativamente mayor $(p=0,03)$ para los alumnos de las escuelas privadas $(47,3 \%)$ que para los de escuela pública (32,1\%). La frecuencia de sobrepeso entre los varones de EPR fue mayor que entre los de la EPU (23,3\% versus $14,3 \%$, $p=0,05)$. Se observó la misma tendencia para las niñas pero sin significación estadística ( $24,0 \%$ versus 17,9\%; $p=0,18)$. Se observó una correlación positiva entre el IMC y el ingreso familiar ( $r=0,14, p=0,04)$. Conclusión: Alrededor del 40\% de los estudiantes encuestados tenían sobrepeso, con mayor frecuencia entre los niños de la escuela privada. Es relevante el desarrollo de estrategias para prevenir la obesidad en todos los niveles de la sociedad, teniendo en cuenta los riesgos potenciales para la salud en la edad adulta.

\section{Palabras clave:}

IMC

Índice de Masa Corporal Adolescentes

Clase Social

\section{Introdução}

A adolescência é caracterizada por intenso crescimento que demanda elevada ingestáo protéico-energética que se não for atendida pode trazer consequências adversas ao crescimento. Por outro lado, durante esse período, as práticas alimentares, muito influenciadas pela mídia e modismos que incentivam o consumo de alimentos de maior densidade energética ${ }^{1}$, associadas à pouca atividade física ${ }^{2}$, podem contribuir para a obesidade, um problema que vem aumentando mundialmente na população jovem.

No Brasil, o índice de sobrepeso e obesidade em crianças e adolescentes de 6 a 18 anos de idade na década de 1970 era de 4\%, triplicando para 13\% no ano de $1997^{3}$. Segundo os dados da Pesquisa de Orçamentos Familiares (POF 2008-2009) ${ }^{4}$, a prevalência de sobrepeso foi de 21,5\% e a de obesidade, de 5,8\% entre adolescentes de 10 a 19 anos. Da mesma maneira, foram observadas prevalências elevadas para sobrepeso e obesidade na Pesquisa Nacional de Saúde do Escolar (PeNSE), realizada pelo IBGE no ano de 2009, com escolares do $9^{\circ}$ ano do ensino fundamental das capitais e Distrito Federal, encontrando-se índice de $16 \%$ de sobrepeso ( $14,5 \%$ na rede pública e $21,4 \%$ na rede privada) e de $7,2 \%$ de obesidade (6,5\% na rede pública e $9,9 \%$ na rede privada). No total, 23,2\% dos escolares analisados apresentavam excesso de peso 5 .

A obesidade tem etiologia multifatorial. A questão genética contribui para aproximadamente $25 \%$ a $30 \%$ dos fatores associados à obesidade ${ }^{6}$. A industrializaçáo tem um papel importante entre os fatores ambientais no quadro atual da obesidade por influenciar no estilo de vida, na alimentação e em outros hábitos da população, por exemplo, na ingestão frequente de fast food, de congelados e de outros alimentos pouco nutritivos e muito calóricos, além do sedentarismo favorecido pelas novas tecnologias ${ }^{7}$.

A crescente preocupação com a ocorrência de obesidade relaciona-se, sobretudo, às comorbidades e complicaçôes geradas pelo excesso de peso. De acordo com os estudos de Abrantes et al. ${ }^{8}$ e Oliveira et al. ${ }^{9}$, cerca de $80 \%$ das crianças e $58 \%$ dos adolescentes obesos tendem a permanecer assim na vida adulta, especialmente se não ocorrer intervenção terapêutica, assim como $70 \%$ dos adolescentes com excesso de peso não tratados na infância tornam-se adultos obesos ${ }^{10}$. Nessa fase da vida tem sido relatada maior ocorrência de doenças cardiovasculares em indivíduos com obesidade prévia em fases precoces da vida. O excesso de peso é a principal causa de resistência à insulina e associa-se fortemente ao diabetes mellitus tipo 2, além de outros problemas de saúde importantes, como alteraçóes ortopédicas, distúrbios respiratórios, hipertensão arterial, dislipidemias e distúrbios psicossociais ${ }^{11}$. Doenças que anteriormente eram manifestadas exclusivamente em adultos, como diabetes mellitus tipo 2 e síndrome metabólica, hoje, justificando o impacto da obesidade ainda em idades precoces, surgem como importantes diagnósticos em crianças e adolescentes.

O diagnóstico precoce da obesidade e sobrepeso durante as primeiras décadas da vida é extremamente relevante para a saúde pública, pois o tratamento é mais eficiente nessa fase da vida e demanda menos custos do estado ${ }^{12}$. Simples programas de intervenção que preconizam dietas saudáveis, prática de atividades físicas e melhores hábitos de vida familiares parecem ser suficientes para alterar o quadro atual, principalmente de sobrepeso.

Assim, o objetivo deste estudo foi comparar a prevalência de excesso de peso em adolescentes de 10 a 14 anos de idade matriculados na rede de ensino pública e particular e correlacionar o índice de massa corporal (IMC) com a renda familiar. 


\section{Métodos}

Estudo transversal com a participação de 213 estudantes do ensino fundamental II de escola pública e de escola privada da cidade de Sorocaba, SP, entre 10 e 14 anos. A seleção das escolas foi feita por conveniência, dentro da região central da cidade, com distância menor de $2 \mathrm{~km}$ entre as escolas selecionadas, condicionada à permissão dos dirigentes. A escola pública abrangia as classes de $5^{\mathrm{a}}$ a $8^{\mathrm{a}}$ séries do ensino fundamental, totalizando 500 alunos na faixa etária de interesse para o estudo matriculados no ano letivo de 2011. A escola particular participante possuía alunos de 5a a 8a séries nos turnos da manhã e da tarde, totalizando 715 estudantes.

Para o cálculo do tamanho amostral utilizou-se a comparação de duas proporçôes, com base no trabalho de Leão et al. ${ }^{13}$ Esse trabalho encontrou prevalência de $8 \%$ de obesidade na escola pública e $30 \%$ na escola particular. Havendo essas duas proporçôes, aplicou-se o teste de hipótese bicaudal com nível de significância de 5\%, poder do teste de 95\%, e verificou-se a necessidade do tamanho amostral mínimo de 80 sujeitos para cada grupo ${ }^{14}$.

Para a seleção da amostra foi realizado sorteio de 150 alunos de cada escola: 84 da escola pública e 129 da escola particular concordaram em participar e retornaram o termo de consentimento livre e esclarecido assinado pelo responsável, totalizando 213 alunos de ambas as escolas.

A classificação social foi obtida através de questionário, segundo critério da Associação Brasileira de Estudos Populacionais (ABEP), de acordo com novo critério $2008^{15}$, que considera existência e quantidade de: televisão, rádio, banheiro, empregada mensalista fixa, DVD, freezer, máquina de lavar roupa, geladeira, automóvel e escolaridade do chefe da casa. A família é enquadrada em um dos nove estratos (A1, A2, B1, B2, C1, C2, D, E), conforme a somatória de pontos. Para fins de análise estatística, reagruparam-se as classes A1 e A2 como nível socioeconômico alto, B1, B2, C1 e C2 como médio e D e E como nível socioeconômico baixo.

A variável renda familiar per capita foi avaliada através de duas perguntas (inclusas no questionário socioeconômico) que consideraram a renda familiar média e o número de pessoas que dependiam dessa renda. O resultado foi calculado em salários mínimos.

O projeto de pesquisa foi aprovado pelo comitê de ética da instituição local sob número 743/2011 e a participação dos alunos só ocorreu mediante concordância do responsável, após assinatura do termo de consentimento livre e esclarecido, conforme determinação 196/96.

Os adolescentes foram pesados em balança antropométrica digital plena, com precisão de $100 \mathrm{~g}$ e capacidade de $150 \mathrm{~kg}$, descalços, vestindo bermudas e camisetas de algodão do uniforme escolar. Foram orientados a permanecer parados em posição ortostática com afastamento lateral dos pés, separados pela plataforma. Para avaliação da estatura foi utilizada uma escala métrica de madeira de 2 metros, subdividida em milímetros, fixada à parede. Uma régua de acrílico foi colocada sob o topo da cabeça do adolescente a fim de se obter um ângulo reto com a parede durante a leitura. Os avaliados foram instruídos a permanecer em posição ereta com a cabeça orientada segundo o plano de Frankfurt, paralelo ao solo, joelhos esticados, pés juntos, braços soltos ao longo do corpo e com tornozelos, glúteos e ombros em contato com a parede. A medida foi efetuada em apnéia inspiratória. Todos os dados antropométricos foram mensurados e anotados pela mesma pesquisadora.

A partir dos dados encontrados, realizou-se o cálculo do índice de massa corporal de cada estudante, através da razão da massa $(\mathrm{kg})$ e estatura elevada ao quadrado $\left(\mathrm{m}^{2}\right)$, sendo $\mathrm{IMC}=$ massa $(\mathrm{kg}) /$ estatura $^{2}\left(\mathrm{~m}^{2}\right)$, analisado de acordo com os gráficos da Organização Mundial da Saúde (OMS) para IMC conforme sexo e idade. Assim, conforme critério da OMS 2007 temos: p 0,1= magreza acentuada, entre p 0,11-3= magreza, entre p 3,1-85= eutrofia, entre p 85,1-97 = sobrepeso, entre $\mathrm{p}>97,1-99,9=$ obesidade e $\mathrm{p}>99,9=$ obesidade grave $^{16}$.

O teste de Kolmogorov foi utilizado para verificar a assimetria dos dados e, de acordo com as variáveis estudadas, foram utilizados a média, o erro padrão da média, o coeficiente de correlação de Spearman, o teste de Mann-Whitney e o $\chi^{2}$. Adotou-se nível de significância de 5\%. As análises foram realizadas com o pacote estatístico SPSS for Windows ${ }^{17}$. 


\section{Resultados}

Dentre os adolescentes avaliados, 40,5\% da EPU e 42,6\% da EPR eram do sexo masculino e 59,5\% da EPU e 57,4\% da EPR eram do sexo feminino, sem diferença estatística entre as escolas $(\mathrm{p}=0,78)$.

As características da populaçáo em estudo, no tocante à idade e aos dados antropométricos nas escolas, encontram-se descritas na Tabela 1.

Tabela 1. Idade e medidas antropométricas dos adolescentes de escola pública e escola privada.

\begin{tabular}{|c|c|c|c|c|}
\hline \multirow{2}{*}{ Variável } & \multicolumn{2}{|c|}{ Escola pública } & \multicolumn{2}{|c|}{ Escola privada } \\
\hline & $\begin{array}{c}\text { Masculino } \\
(n=34)\end{array}$ & $\begin{array}{l}\text { Feminino } \\
(\mathrm{n}=50)\end{array}$ & $\begin{array}{c}\text { Masculino } \\
(n=55)\end{array}$ & $\begin{array}{c}\text { Feminino } \\
(n=74)\end{array}$ \\
\hline Idade (anos) & $12,7 \pm 0,17$ & $12,4 \pm 0,15$ & $11,4 \pm 0,14^{*+}$ & $11,8 \pm 0,11$ \\
\hline Peso (kg) & $53,1 \pm 2,07$ & $49,3 \pm 1,42$ & $48,9 \pm 2,04$ & $49,6 \pm 1,42$ \\
\hline Estatura (m) & $1,60 \pm 0,01$ & $1,56 \pm 0,01$ & $1,52 \pm 0,01^{+}$ & $1,54 \pm 0,01$ \\
\hline IMC $\left(\mathrm{kg} / \mathrm{m}^{2}\right)$ & $20,6 \pm 0,70$ & $20,3 \pm 0,51$ & $20,8 \pm 0,65$ & $20,8 \pm 0,43$ \\
\hline
\end{tabular}

IMC: índice de massa corporal; valores média \pm erro padrão da média. ${ }^{\star} \mathrm{p}<0,05$ masculino versus feminino; ${ }^{+} \mathrm{p}<0,05$ escola pública versus escola privada.

Verificou-se que na EPU 65,5\% dos adolescentes eram eutróficos, 2,4\% apresentavam baixo peso, 12\%, sobrepeso e $20,2 \%$ eram obesos. Na EPR, 51,2\% eram eutróficos, $1,6 \%$ apresentavam baixo peso, 18,6\%, sobrepeso e $28,7 \%$ eram obesos, os mesmos diagnósticos nutricionais, não se observando diferença entre os sexos.

Analisando-se as classificaçôes de sobrepeso e obesidade agrupadas, verificou-se prevalência global de excesso de peso na amostra estudada (Tabela 2) de 41,3\%. Nos meninos foi de 47,2\% e, nas meninas, de 37,1\%. Na comparaçáo dos resultados isolados, a frequência de excesso de peso foi maior para os alunos da escola particular do que para os da escola pública, com significância estatística $(\mathrm{p}=0,03)$.

Tabela 2. Prevalência de excesso de peso (sobrepeso e obesidade) segundo o tipo de escola pesquisada.

\begin{tabular}{lccc}
\hline \multicolumn{1}{c}{ Prevalência de excesso de peso } & $\mathrm{n}$ & $\mathrm{N}$ & \% \\
\hline Alunos da escola pública & 27 & 129 & 32,1 \\
Alunos da escola particular & 61 & 213 & 47,3 \\
Total de alunos & 88 & 41,3
\end{tabular}

n=número total de excesso de peso; N=número total de alunos.

Comparando-se sexo e escolas (Figura 1), observou-se maior frequência de excesso de peso entre os meninos da EPR do que entre os da $\operatorname{EPU}(23,3 \%$ versus $14,3 \% ; \mathrm{p}=0,05)$. Com relação ao grupo das meninas, não houve diferença estatística nas frequências de excesso de peso entre EPR e EPU $(24,0 \%$ versus $17,9 \%$; $\mathrm{p}=0,18)$. 




Figura 1. Estado nutricional segundo o índice de massa corporal dos adolescentes de escola pública e particular conforme sexo.

A renda familiar média per capita dos alunos da escola particular foi de 1,92 $\pm 1,5$ salário mínimo, significativamente superior $(\mathrm{p}<0,01)$ à dos alunos da escola pública, que correspondeu a 1,36 $\pm 1,1$ salário mínimo.

Houve discreta correlação positiva entre IMC e renda familiar $(\mathrm{r}=0,14, \mathrm{p}=0,04$ - Figura 2). Não houve correlação entre IMC e idade dos adolescentes ( $\mathrm{p}=0,11)$, nem entre o número de aparelhos de TV ( $\mathrm{p}=0,45)$, DVD ( $\mathrm{p}=0,18)$ ou automóveis referidos no domicílio $(\mathrm{p}=0,60)$.

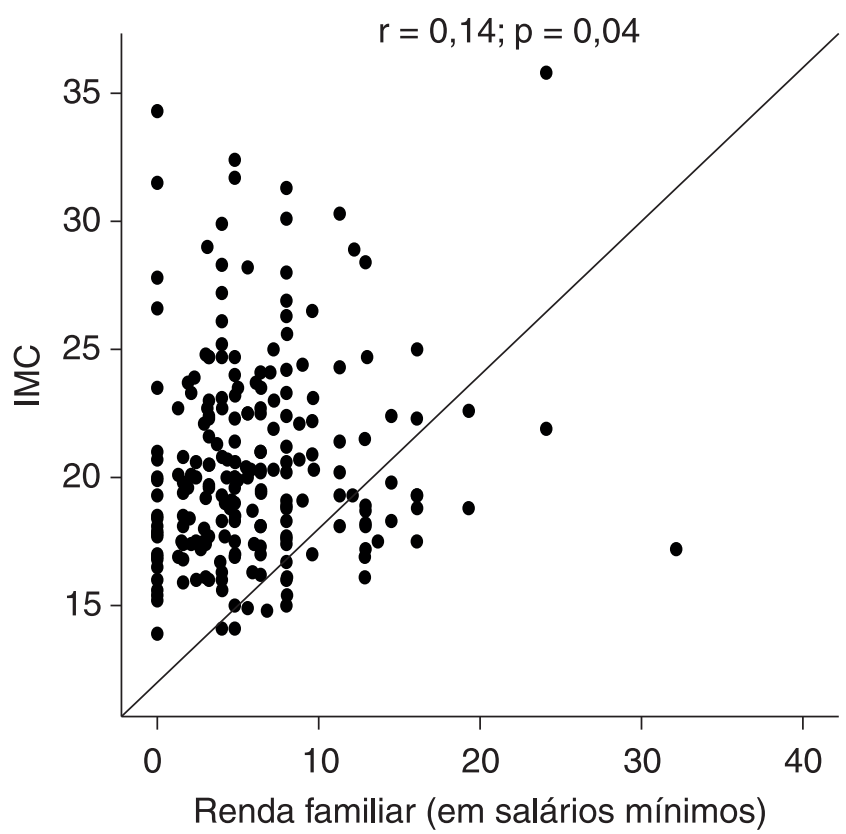

Figura 2. Correlação entre IMC e renda familiar dos entrevistados. 


\section{Discussão}

Este estudo demonstrou elevada prevalência de excesso de peso nos adolescentes do ensino fundamental, sobretudo os pertencentes à escola privada. Os resultados apontaram também que o IMC associou-se positivamente com a renda familiar, em concordância com estudos recentes. Tendo em vista o preocupante avanço da prevalência do excesso de peso é preciso adotar medidas preventivas em todos os níveis da sociedade; na família, com a escolha saudável de alimentos e dedicaçáo de tempo às atividades físicas; na escola, com o cuidado com os lanches hipercalóricos e o consumo abusivo de refrigerantes; e na sociedade, com campanhas de promoção à saúde que busquem reduzir comorbidades futuras. Estudo realizado por Baker et al. ${ }^{18}$ revelou aumento significativo do risco de doença coronariana na vida adulta para cada unidade acrescida no IMC. A obesidade grave ou persistência de excesso de peso por período superior a 3 anos, segundo Pacheco Torres et al. ${ }^{19}$, relaciona-se com um risco 48\% maior de desenvolvimento de esteatose hepática não alcoólica em crianças.

Neste estudo, a frequência de sobrepeso/obesidade nos adolescentes de ambas as escolas foi de 41,3\%; nos meninos foi de $47,2 \%$ e, nas meninas, de $37,1 \%$. Esse resultado foi superior ao encontrado em trabalho recente ${ }^{20}$, que constatou a prevalência de excesso de peso em 35,7\% para meninos e 26,2\% para meninas. Dados recentes do Sistema de Vigilância de Fatores de Risco e Proteção para Doenças Crônicas por Inquérito Telefônico (Vigitel) ${ }^{21}$ referentes a 2011 demonstram um grau de obesidade em adultos entre 12,5\% (Palmas) e 21,4\% (Macapá). Já o excesso de peso variou de 39,8\% (São Luís) a 55,4\% (Porto Alegre). Esses dados condizem com a realidade encontrada no presente estudo, demonstrando uma preocupante elevaçáo da prevalência de excesso de peso no país. De fato, a prevalência de excesso de peso saltou de 4\%, na década de $1970^{3}$, para quase $50 \%$ em adolescentes.

Os adolescentes da escola privada apresentaram prevalência de excesso de peso significativamente maior do que os da escola pública. Resultados similares foram encontrados também nos estudos de Suñé et al. ${ }^{22}$ e Vieira et al. ${ }^{23}$ Porém nos resultados do presente estudo foi possível constatar prevalências ainda maiores, tanto na escola pública quanto na particular. Em estudo realizado no município de Capão da Canoa, RS ${ }^{22}$, que avaliou 510 adolescentes entre 11 e 13 anos de idade das redes de ensino pública e privada, foram verificadas prevalências similares em meninos e meninas (27,9\% versus $21,6 \%)$. Quanto ao tipo de escola, a prevalência de sobrepeso em adolescentes do ensino público foi de $23,1 \%$. Em adolescentes do ensino privado, essa prevalência mostrou-se superior (39,2\%), porém ainda inferior aos 47,3\% encontrados no estudo.

No estudo de Vieira et al. ${ }^{23}$, verificou-se que dentre escolares das redes de ensino público e privado do município de Pelotas, RS, na faixa etária de 11 a 17 anos, a prevalência de sobrepeso nos meninos foi de 14,4\% e, nas meninas, de 18,5\%. Esse estudo também encontrou maior prevalência de excesso de peso em adolescentes da rede particular de ensino. Além disso, nessa mesma pesquisa, os autores acreditam que os alunos da rede privada estejam mais expostos a fatores que contribuem para o excesso de peso, como o uso de aparelhos eletrônicos (celulares, videogames, computadores), deslocamentos em carro, consumo de alimentos industrializados, dentre outros.

Destaca-se ainda, nesta pesquisa, que a prevalência de excesso de peso esteve mais elevada entre os meninos. Segundo a literatura, enquanto meninos buscam ganho de peso por meio do aumento da massa muscular, meninas visam corpos mais magros, provavelmente mais influenciadas pelo padrão de beleza exaltado pelos meios de comunicação ${ }^{24,25}$. Nesse contexto, Frisancho ${ }^{26}$ sugere que o IMC é um indicador de massa corporal total e não de massa de gordura total; assim, é importante destacar que o índice de massa corporal engloba também massa magra, o que pode contribuir para a prevalência maior de excesso de peso em meninos.

A frequência geral de baixo peso no presente estudo $(2,4 \%)$ foi menor em comparação a outros estudos. Na Pesquisa Nacional sobre Saúde e Nutriçáo (PNSN) verificou-se que, no município do Rio de Janeiro, 7,3\% dos escolares apresentavam baixo peso ${ }^{27}$. Pereira e Veiga ${ }^{28}$, em Cuiabá, Mato Grosso, encontraram 19,1\% de adolescentes com IMC abaixo do percentil 10, enquanto Pereira ${ }^{29}$, em estudo com adolescentes no município do Rio de Janeiro, encontrou baixo peso em $13,0 \%$ das meninas e em 10,0\% dos meninos. Por outro lado, estudo de Toral et al. ${ }^{30}$ encontrou um percentual ainda menor que o do presente estudo em adolescentes com déficit de altura para idade $(1,8 \%)$. Esse quadro, que está de acordo com o observado no presente estudo, indica a transição do desequilíbrio energético da carência para o excesso nos adolescentes brasileiros.

Outro dado interessante encontrado no presente estudo foi a discreta correlaçáo positiva entre IMC e renda familiar. Estudo de Neutzling et al. ${ }^{31}$ encontrou uma associação direta entre nível socioeconômico e obesidade na adolescência. Avaliando os dados da PNSN, esses autores verificaram em adolescentes pertencentes a famílias com renda familiar per capita baixa, intermediária e alta, prevalência de sobrepeso de 5,6\%, 8,4\% e 15,9\%, respectivamente. Dois estudos recentes ${ }^{32,33}$, divulgados 
em 2011, também constataram que no Brasil há um alto índice de sobrepeso vinculado às classes socioeconômicas mais elevadas. Na pesquisa de orçamento familiar do Instituto Brasileiro de Geografia e Estatística (IBGE) de 2009 observou-se que a prevalência de excesso de peso aumenta conforme a elevação da renda ${ }^{4}$. No presente estudo, esta correlação entre IMC e renda familiar foi fraca, provavelmente pela amostragem de conveniência escolhida.

Algumas limitações deste estudo devem ser consideradas. A natureza transversal dos dados não permite estabelecer associação causal entre as variáveis. Outra limitação é a utilização de questionário, que confere caráter subjetivo ao estudo. Por outro lado, estudos transversais são importantes para o direcionamento de futuros estudos prospectivos.

Em síntese, o que mais chamou a atenção no presente estudo foi o significativo excesso de peso, particularmente entre os adolescentes que frequentavam a escola privada, e também relevante naqueles que frequentavam a escola pública. Esse fato está em consonância com o fenômeno da transição nutricional que vem sendo observado no Brasil: os problemas relacionados ao excesso de gordura corporal aumentaram significativamente nas últimas décadas. Levando-se em consideração a alta probabilidade de a obesidade da adolescência permanecer na vida adulta, ocasionando efeitos adversos à saúde, é importante ampliar os estudos sobre essa faixa etária, visando avaliar a dimensão do problema e criar estratégias de prevençáo e controle. Deve-se ressaltar o importante papel da escola na implementaçáo de programas educacionais que procurem encorajar a atividade física e os hábitos alimentares adequados. É necessário o envolvimento de pais, professores, alunos e de toda a sociedade nessa discussão, visando desacelerar esse preocupante quadro de aumento da prevalência de excesso de peso na adolescência.

\section{Referências}

1. Zaida G. A criança, os comerciais de televisão e a cultura alimentar: uma análise crítica. Educ Soc. 1992; 43: 505-21.

2. Silva RCR, Malina RM. Nível de atividade física em adolescentes do Município de Niterói, Rio de Janeiro, Brasil. Cad Saúde Pública. $2000 ; 16(4): 1091-7$. http://dx.doi.org/10.1590/S0102-311X2000000400027

3. World Health Organization, International Diabetes Federation. Fight childhood obesity to help prevent diabetes. WHO; 2005. Available from: http:// www.who.int/mediacentre/news/releases/2004/pr81/en/index.html.

4. Instituto Brasileiro de Geografia e Estatística - IBGE. Pesquisa de Orçamento familiar 2008-2009: antropometria e análise do estado nutricional de crianças, adolescentes e adultos no Brasil. Rio de Janeiro: IBGE; 2010.

5. Instituto Brasileiro de Geografia e Estatística - IBGE. Pesquisa nacional de saúde do escolar. Rio de Janeiro: Ministério do Planejamento, Orçamento e Gestão; 2009.

6. Frisancho AR. Prenatal compared with parental origins of adolescent fatness. Am J Clin Nutr 2000; 72: 1186-90.

7. Damiani D, Damiani D, Oliveira RG. Obesidade: fatores genéticos ou ambientais? Pediatr Mod. 2002; 38(3): 57-80.

8. Abrantes MM, Lamounier JA, Colosimo EA. Prevalência de sobrepeso e obesidade em crianças e adolescentes das regiões Sudeste e Nordeste. J Pediatr. 2002; 78(4): 335-40. http://dx.doi.org/10.1590/S0021-75572002000400014

9. Oliveira AMA, Oliveira AC, Almeida MS, Almeida FS, Ferreira JBC, Silva CEP, et al. Fatores ambientais e antropométricos associados à hipertensão arterial infantil. Arq Bras Endocrinol Metab. 2004; 48(6): 849-54. http://dx.doi.org/10.1590/S0004-27302004000600011

10. Vande Weyer M, Bolterys S, Guzman E. L'approche pluridisciplinaire de l'enfant obèse à l'Internat Diététique de "Clairs Vallons". Rev Med Brux. 2005; 26: S215-8.

11. Monteiro P, Victora C, Barros F. Fatores de risco sociais, familiares e comportamentais para obesidade em adolescentes. Rev Panam Salud Publica. 2004; 16(4): 250-8. http://dx.doi.org/10.1590/S1020-49892004001000004

12. Saldiva SRD, Escuder MML, Venâncio SI, Benicio D’Aquino MH. Prevalence of obesity in preschool children from five towns in São Paulo State, Brazil. Cad Saúde Pública 2004; 20(6): 1627-32. http://dx.doi.org/10.1590/S0102-311X2004000600021

13. Leão LS, Araujo LM, Moraes LT, Assis AM. Prevalência de obesidade em escolares de Salvador, Bahia. Arq Bras Endocrinol Metab 2003; 47 : $151-7$. http://dx.doi.org/10.1590/S0004-27302003000200007

14. Laboratório de Epidemiologia e Estatística - Lee. São Paulo: Cálculo do tamanho amostral. [acesso em 2011 mar.]. Disponível em: http://www.lee. dante.br/pesquisa/amostragem/amostra.html

15. Associação Brasileira de Estudos Populacionais - ABEP. O novo critério padrão de classificação econômica Brasil [Internet]. Disponível em: http:// www.viverbem.fmb.unesp.br/docs/classificaçãobrasil.pdf

16. Organização Mundial da Saúde - OMS. OMS growth charts 2007 [Internet]. Disponível em: http://www.189,28.128/nutricao/docs/geral/sisvan_ norma_tecnica_criancas.pdf

17. SPSS for Windows. release 12.0.0 [computer program]. Chicago: SPSS; 2003. 
18. Baker JL, Olsen LW, Sørensen TIA, Sci M. Childhood bodymass index and the risk of coronary heart disease in adulthood. $N$ Engl $J$ Med. 2007; 357(23): 2329-37. http://dx.doi.org/10.1056/NEJMoa072515

19. Torres LP, Lamas RP, Arbelo TF, Alonso MCV, Martinez R. Hígado graso no alcohólico en niños obesos. Rev Cuba Pediatr. 2006; 78(1).

20. Peres SV, Latorre MRDO, Slater B, Tanaka LF, Silva MVS. Prevalência de excesso de peso e seus fatores associados em adolescentes da rede de ensino público de Piracicaba, São Paulo. Rev Paul Pediatr. 2012; 30(1): 57-64.

21. Portal da Saúde. Vigitel 2011 [Internet]. Disponível em: http://portal.saude.gov.br/portal/saude/profissional/area.cfm?id_area=1521

22. Suñé FR, Dias-da-Costa JS, Olinto MT, Pattussi MP. Prevalence of overweight and obesity and associated factors among schoolchildren in a southern Brazilian city. Cad Saude Publica 2007; 23: 1361-71.

23. Vieira MF, Araújo CL, Hallal PC, Madruga SW, Neutzling MB, Matijasevich A, et al. Nutritional status of first to fourth-grade students of urban schools in Pelotas, Rio Grande do Sul State, Brazil. Cad Saude Publica 2008; 24: 1667-74.

24. Vilela JE, Lamounier JA, Oliveira RG, Ribeiro RQ, Gomes EL, Barros Neto JR. Assesment of eating attitudes of children and adolescents in Belo Horizonte City. Psiquiatr Biol. 2001; 9: 121-30.

25. Conti MA, Costa LS, Peres SV, Toral N. The body dissatisfaction of adolescents: an exploratory research. Physis. 2009; $19: 509-28$.

26. Frisancho AR. Anthropometric Standards for the assessment of growth and nutritional status. Ann Arbor: University of Michigan Press; 1990.

27. Rio de Janeiro. Secretaria Municipal de Saúde. Secretaria Municipal de Educação. II Pesquisa de Saúde e Nutrição em Escolares. Rio de Janeiro; 2000. n. 2, p. $1-4$.

28. Pereira SF, Veiga GV. Avaliação do estado nutricional de adolescentes estudantes das Escolas Municipais de Cuiabá - MT. Pediatr Mod. 1998; 34(6): 279-90.

29. Pereira RA. Avaliação antropométrica do estado nutricional. In: Sichieri R, editor. Epidemiologia da obesidade. Rio de Janeiro: UERJ; 1998. p. 43-64.

30. Toral N, Slater B, Silva MV. Consumo alimentar e excesso de peso de adolescentes de Piracicaba, São Paulo. Rev. Nutr. 2007; 20(5): 449-459.

31. Neutzling M, Taddei JA, Rodrigues EM, Sigulem DM. Overweight and obesity in Brazilian adolescents. Int J Obes. 2000; 24(7): 869-74.

32. Guedes DP, Rocha GD, Silva AJRM, Carvalhal IM, Coelho EM. Effects of social and environmental determinants on overweight and obesity among Brazilian schoolchildren from a developing region. Rev Panam Salud Publica. 2011; 30(4): 295-302.

33. Fernandes RA, Christofaro DG, Freitas Junior IF, Cardoso R, Kawaguti SS, Oliveira AR, et al. Socioeconomic status as determinant of risk factors for overweight in adolescents. Ciênc Saúde Coletiva. 2011; 16(10): 4051-4057. 